\title{
Multi-agent-Based Voltage Cooperative Control in Smart Grids
}

\author{
Sun Jian ${ }^{\mathrm{a},{ }^{*}, \text { Wang Qiang }}{ }^{\mathrm{b}}$ \\ College of Electrical Engineering \& New Energy, China Three Gorges University, Yichang, China \\ aemail: sunjian2009@ctgu.edu.cn, ${ }^{b}$ email:wq00518@sohu.com
}

\begin{abstract}
keywords: Multi-Agent; Voltage Cooperative Control; Smart Grids.
Abstract. To low transmission power loss, maintain voltage stability and improve voltage profile, a novel multi-agent-based voltage control scheme is proposed in this paper by dynamically adapt configuration of control devices, including the automatic voltage regulator of generator, transformer with under-load tap changer, and flexible alternating current transmission systems(FACTS). Multi-agent system is designed with consideration of the technical constraints and load dynamic model effect. Information exchange among agents is facilitated by Peer-to-peer device. The proposed scheme coordinates the operation of installed voltage control devices on both low voltage and high voltage levels. A simulation test model is implemented using a parallel computing arrangement for a 30-bus standard test system to evaluate the proposed voltage control scheme.
\end{abstract}

\section{Introduction}

Large interconnected power systems throughout the world are frequently subjected to widespread blackouts, which interrupt millions of consumers and cost billions of dollars. The most severe interruptions occur in highly loaded interconnected power systems because of high voltage (HV) line disruption followed by multiple contingencies[1]. Power system behavior in an emergency state is characterized by complex interaction among control devices (voltage controllers). The latter usually uses local signals only and does not coordinate their actions with one another. Absence of coordination is the shortcoming of the existing protection system and it may lead to voltage collapse and blackout. An agent is a computer system that is capable of autonomous action in some environment to meet its design objectives[2,3,4,5].

MACS have been realized as operable control systems in power system[6]. MACS is a decentralized control system approach, which has many applications in power systems, e.g., decentralized secondary voltage control was initiated by the French electricity company (EDF) and followed by some other electricity utilities in European countries about three decades ago [7]. With the development of the open electricity market, great efforts have been made to improve the performance of secondary voltage control schemes[8].

In this paper, a new MACS-based voltage cooperative control is proposed for smart grids. The algorithm dynamically adapts the settings of control devices, such as AVR of generators, ULTC, and FACTS, to optimize certain objectives. In this work, communication of peer-to-peer device coordination is developed to facilitate information exchange among agents. A simulation test model is implemented to test the control effects of reducing transmission power loss, maintaining voltage stability, and improving voltage profile.

\section{Proposed Multi-agent-Based Strategy}

Agents of one multi-agent system are located in the same logical network (homogeneous). For the establishment of a connection in a static homogeneous network, the following conditions must be satisfied[8]:

- Knowledge of the IP addresses of the agents; and

- Knowledge of the agent framework's communication port.

According to the electric component to which an agent is associated, the agents in the MACS can be classified into generator agents (GAs) and load agents. Each agent has its specific rules for 
performing its functions. In the proposed MACS, there is no central controller or coordinator. Each agent works autonomously and independently based on the information it receives from other agents. Thus, the MACS works in a completely decentralized manner. Load agents are classified into the following three categories:

- Transmission agent (TA), installed in TL at one of its ends, will control transmission transformer tap changers;

- Supported load bus agent (SLBA), installed on load buses that have reactive power compensators, such as static compensator (STATCOM), unified power-flow controller (UPFC), or capacitor banks, in addition to a ULTC; SLBA will control the reactive power compensators and the ULTC; and

- Load bus agent (LBA), installed on buses that have a ULTC only; LBA will control only this ULTC.

GAs, TAs, and SLBAs are called supporting agents (SAs). The agent always has the following set of data:

- Local state variables: primary and secondary voltage, and line power flows; and

- Operating characteristics of the local equipment: tap range of the tap changer, excitation current of the generator (If), STATCOM capacity, number of capacitors banks stages.

Any agent has two goals:

- The local goal is to maintain local state variables and equipment operating characteristics within the normal range; and

- a global goal of preventing voltage collapse or violations.

\section{Agent Behavior}

Generator Agent. Generator voltage is controlled by AVR and transformer tap changer (TC). GA obtains local information about the $I_{f}$ of the generator, primary and secondary voltages at the generating substation, line power flows, and transformer tap ranges.

The generator $I_{f}$ is estimated as in [8]. The GA applies the following rules:

1. $I_{f}$ it receives an RM from a violated-voltage LBA to increase $Q_{g}$, it will reply an agree message (AM) to the sender agent if $\mathrm{VH}$ is less than $1.1 \mathrm{p}$.u, if the If of its generator is less than $95 \%$ of $I_{f}$, max, and if neighboring line flows are less than $95 \%$ of maximum capacity.

2. If during increasing $\mathrm{Qg}, \mathrm{VH}$ becomes more than $1.1 \mathrm{p}$.u or If becomes more than $95 \%$ of $I_{f}$, max, or $\mathrm{S}_{1}$ of a neighboring line becomes more than $95 \%$ of $\mathrm{S}_{1}$; max, it sends an IM to the violated-voltage LBA telling its inability to increase $Q_{g}$ anymore.

3. If If exceeds $I_{f}$; max, it sends an RM to SA.

Load Agent. The autonomous control manner of a load bus equipped with a shunt static compensator and ULTC. When an SLBA receives an RM, it checks operating characteristics at its local substation before replying. If it agrees, it increases RPS by increasing the $V_{\text {ref }}$ of the local compensator according to the algorithm. If an SLBA or LBA needs support, it sends an RM to an SA according to the algorithm.

Simulation Model Implementation. MACS and power system are usually simulated on the same PC and also use single-threaded programming environments. So, they cannot execute two or more programs in parallel and also peer-to-peer message transfer is not possible.

Therefore, a multithread platform that allows research of real performance and communication bandwidth requirements of the MACS is a must. An agent platform provides a basis for the implementation of a MACS and the means to manage agent execution and message passing. Among available platforms, JADE is selected because of its good capabilities and logical structure. JADE is an open source software, and it is the most FIPA (an international standard for agent interoperability) compliant platform. In this study, Java is the programming language of choice because of its multithreading capability and its object-oriented technology.

Voltage stability is largely affected by load characteristics. Three different load models are considered in this paper: the ZIP load model, the voltage-dependent load model, and Jimma's load model.Each load type has a specific mathematical model for the active power component and the reactive power component as given in Overbye and Milosevic . 
Power system is implemented on MATLAB/PSAT. Because JADE is selected as a platform for MACS development, an interface for linking MATLAB/PSAT with JADE is designed.

Data agents, which are Java objects that contain different data structures, are used. Two main subroutines, GetStatus and SetStatus, were constructed in Java language for reading status from the power system and also setting control actions to it by data agents. During time domain simulation, information about power system operating conditions at each integration step passes from the MATLAB environment to JADE by data agents. Then, agents inside the JADE environment process this information, produce control actions if needed, and put information about control actions inside data agents to carry to the MATLAB environment.

The case study power system used to evaluate the proposed MACS is the modified IEEE 30-bus test system shown in Fig. 1. Parameters of the IEEE 30-bus test system are found in [8], and parameters of the modified IEEE 30-bus test system are given in IEEE (2013). The system is equipped with STATCOM and UPFC. Their optimal placement is determined by continuation power-flow analysis.

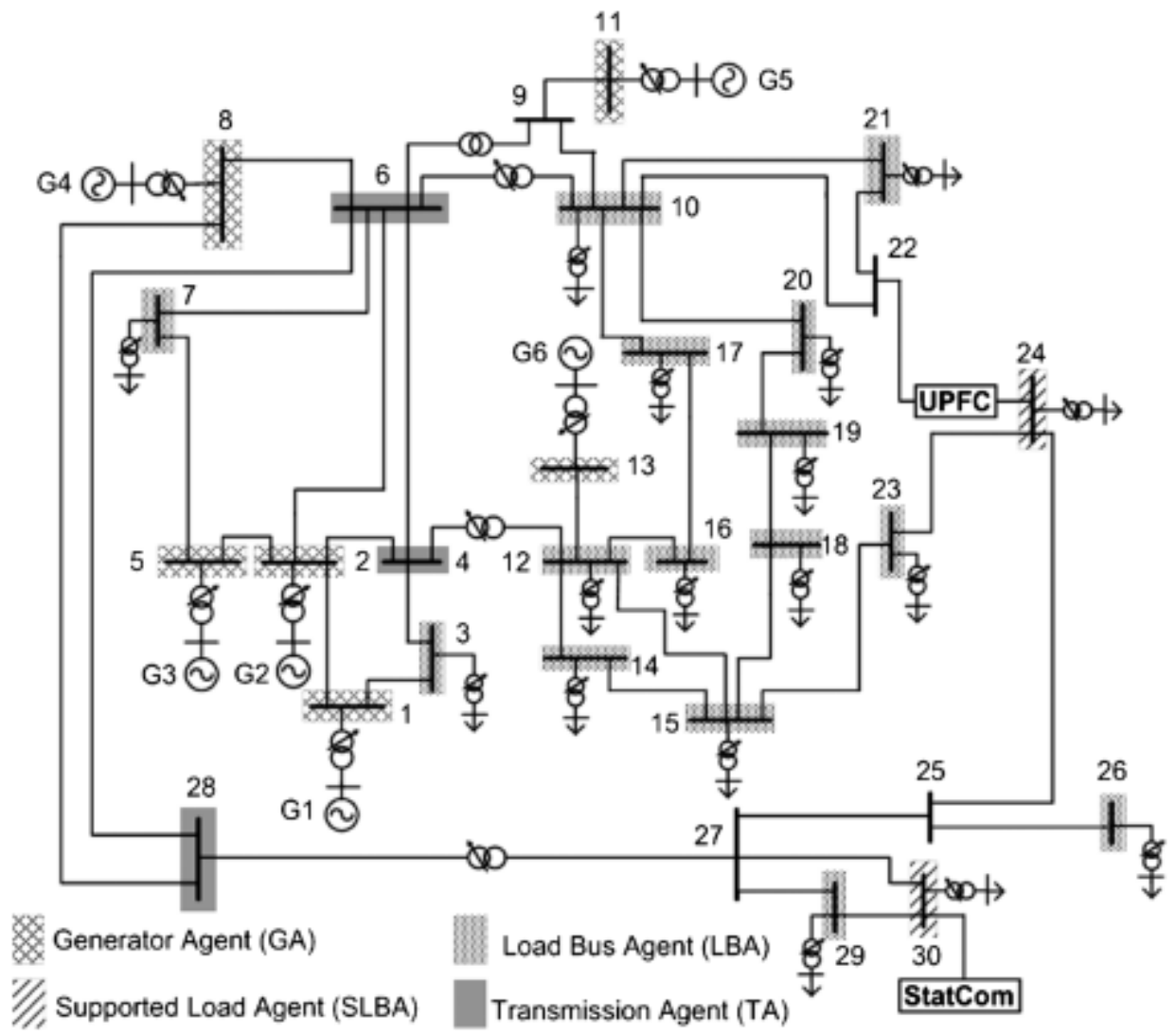

Fig. 1. Modified IEEE 30-bus test system

The weakest buses are 30,29,26, 24, and 19. The optimal locations of FACT devices are nearby these buses. Therefore, STATCOM is installed at Bus 30 to also support voltage at Buses 29 and 26. UPFC is installed in series to TL 24-22 near to Bus 24.

The following modifications are made to the IEEE 30-bus test system (IEEE 2013):

- STATCOM and UPFC are installed as already mentioned. Maximum currents of STATCOM and UPFC are 1 and 1.5 p.u, respectively;

- ULTCs are installed between the subtransmission system and distribution system loads; ULTC tap range is _12 steps;

- Transmission transformers at Bus 4, Bus 6, and Bus 28 are equipped with ULTCs whose ULTC tap range is _14 steps;

- ULTC time delay for the first tap movement is $15 \mathrm{~s}$, and the ULTC time delay for subsequent tap movements is $5 \mathrm{~s}$;

- Each generator is modeled by a sixth-order dynamic model and is equipped with a Type II turbine governor and Type II AVR; 
- All generators are equipped with over-excitation limiters (OXLs) ; and

- OXLs maximum field currents for generators G1, G2, G3, G4, G5, and G6 are 3, 3, 1.6, 3, 3, and 1.5 p.u, respectively. Each load is modeled by three different models as follows:

1. A ZIP load with 33\% constant impedance, 33\% constant current, and 34\% constant power for both active and reactive components.

2. A $100 \%$ voltage-dependent load for both active and reactive power components and voltage exponents for the active and reactive powers of 2.3 and 2.2, respectively.

3. Jimma's load starting with 33\% constant impedance, 33\% constant current, $34 \%$ constant power for both active and reactive components, and coefficient of voltage magnitude time derivative $\mathrm{KV}=$ 20 .

\section{Test Results}

A representative test cases are presented to demonstrate different modes of operation of the proposed MACS comparing it with the conventional decentralized voltage control approach[6] and MACS of voltage control [7].

At $2 \mathrm{~s}$ of simulation, a large sudden increase occurs simultaneously to the loads at Bus 7 and Bus 26, for all load models, as shown in Table 1, and the TL between Bus 5 and Bus 7 is disconnected.

The result reveals the voltage variation of $\mathrm{HV}$ buses on applying the conventional control. After load increase and the TL disconnection, voltages at Bus 7 and Bus 26 fall below the 0.95 p.u constraint for all load models. Voltage on the low voltage (LV) side only of the same buses returns fast to the 0.95 p.u limit by the action of the ULTC connecting HV and LV sides.

Table 1. Load changes at $2 \mathrm{~s}$

\begin{tabular}{lllll}
\hline Load & \multicolumn{2}{c}{ Time $<2 \mathrm{~s}$} & \multicolumn{2}{c}{ Time $>2 \mathrm{~s}$} \\
\hline Bus 7 & $22.8 \mathrm{MW}$ & $10.9 \mathrm{MVar}$ & $100.3 \mathrm{MW}$ & 48MVar \\
Bus 26 & $2.2 \mathrm{MW}$ & $0.7 \mathrm{MVar}$ & $8.3 \mathrm{MW}$ & $2.61 \mathrm{MVar}$ \\
\hline
\end{tabular}

On the other hand, when the proposed MACS is applied, all bus voltages are kept within 0.95 p.u and 1.1 p.u . The $I_{f}$ for all generators are kept under the thermal limit for both control schemes. The voltage behavior for the ZIP and voltage-dependent load models are similar. It differs for Jimma's load model because its reactive power depends on the time derivative of the bus voltage. This property opposes the voltage correction attempted by the control scheme.

MACS reports the following actions: For the case of ZIP and voltage-dependent load models, LBA at Bus 7 and Bus 26 detect the voltage violation at approximately $2 \mathrm{~s}$ of simulation. The LBA at Bus 7 sends an RM to GA at G4, which has the highest relative dV/dQ. It replies with an AM to increase RPS by increasing its $V_{\text {ref }}$ (secondary voltage control) as shown in Table 2. For LBA at Bus 26, it sends an RM to SA at Bus 24 (UPFC), which has the highest relative dV/dQ to increase RPS. The chosen SA replies with an AM to increase RPS by increasing its $V_{\text {ref }}$ (secondary voltage control) as shown Table 2.

Table 2. Changes of $V_{\text {ref }}$ of involved supporting devices

\begin{tabular}{llll}
\hline Device & \multicolumn{2}{c}{$\square V_{\text {ref }}(\mathrm{p} . \mathrm{u})$} \\
\cline { 2 - 4 } & Zip load model & $\begin{array}{l}\text { Voltage-dependent } \\
\text { load model }\end{array}$ & Jimma's load model \\
G1 & 0.04 & 0.02 & 0.02 \\
G2 & 0.077 & 0.077 & 0.077 \\
G4 & 0.05 & 0.05 & 0.05 \\
G5 & 0.03 & 0.0 & 0.0 \\
UPFC & 0.01 & 0.01 & 0.01 \\
STATCOM & 0.01 & 0.01 & 0.01 \\
Transmission ULTC at & 0.03 & 0.02 & 0.06 \\
Bus 28 & & \\
\hline
\end{tabular}


At approximately $21 \mathrm{~s}$, LBA at Bus 26 detects that voltage violation is eliminated successfully. Hence, it sends an IM to the working SA at Bus 28 to stop increasing its tap ratio. At approximately $24 \mathrm{~s}, \mathrm{GA}$ at G4 detects that the power flow of the neighboring line between Bus 6 and Bus 8 is approaching the maximum capacity. Therefore, it sends an IM to LBA at violated-voltage Bus 7 telling that it cannot increase RPS. Hence, LBA at Bus 7 sends an RM to SA at Bus 2, which has the second highest relative dV/dQ. The chosen SA replies with an AM to increase RPS by increasing its $V_{\text {ref }}$ as shown in Table 2. For the Jimma's load model case, the scenario is similar except that LBA at Bus 7 and Bus 26 start to detect the voltage violation later at approximately 4 and $6 \mathrm{~s}$, respectively. Further, LBA at theses buses had to wait until about 70 and $46 \mathrm{~s}$, respectively, to make sure that the voltage at their corresponding buses recovers to the 0.95 p.u limit and sends IM to SAs.

\section{Conclusion}

A new MACS-based voltage control scheme is proposed. The algorithm dynamically adapts the settings of control devices to control the generator excitation current, maintain voltage stability, and improve the voltage profile on both LV and HV nodes. The constraint is to keep the technical capability limits of system equipment and, in particular, the TL power-flow limits. The proposed MACS coordinates the operation of many types of voltage control devices widely scattered over the power grid, including transformer ULTC, FACTS devices, generator AVR, and TL tap changer. Decentralized design of the MACS is adopted such that every agent can communicate with all other agents to facilitate information exchange and accelerate control decision making. A simulation test model is implemented using a parallel MATLB-JADE arrangement for a modified IEEE 30-bus test system (IEEE 2013). The system is examined for different load models under different emergency conditions. The performance of the proposed MACS is compared with the conventional method and a recent MACS-based approach for voltage control. Results confirm the efficacy and superiority of the proposed system under all examined conditions.

\section{Acknowledgement}

This work is supported by Yichang Council Science and Technology Bureau(A2012-302-14 and A2012-302-15).

\section{References}

[1] International Council on Large Electric Systems (CIGRE), Defense plan against extreme contingencies. CIGRE Task Force C2.02.24, France.

[2] Elmitwally, A., Voltage control strategy for distribution systems with flexible DG. Int. J. Distrib. Energy Resour., 6(4), 359-373.

[3] Gehao, S., Xiuceng, J., and Yi, Z., Optimal coordination for multi-agent based secondary voltage control in power system. IEEE/Power Engineering Society (PES) Transmission and Distribution Conf. Exhibition, IEEE, New York, NY.

[4] Llorez, J., Tapia, A., Criado, R., and Grijalba, J., Secondary voltage control based on a robust multivariable PI controller.Proc., $11^{\text {th }}$ Power Systems Computation Conf. (PSCC), PSCC board, Avignon, France, 1011-1018.

[5] Wang, H., Multi-agent co-ordination for the secondary voltage control in power system contingencies.IET Gener. Transm. Distrib., 148(1), 61-66.

[6] Panasetsky, D., and Voropai, N., A multi-agent approach to coordination of different emergency control devices against voltage collapse. Proc., IEEE PowerTech Int. Conf., IEEE, New York, [7]NY.Keskin, M., Continuation power flow and voltage stability in power systems. Master thesis, Middle East Technical Univ., Turkey.

[8] Rohbogner, G., Fey, S., Benoit, P., Wittwer, C., and Christ, A., Design of a multiagent-based voltage control system in peer-to-peer networks for smart grids. Energy Technol., 2(1), 107-120. 